\title{
Erratum to: Exploring correlation for fast skyline computation
}

\author{
Boseon Yu' ${ }^{1}$ Wonik Choi ${ }^{2} \cdot \operatorname{Ling~Liu}^{3}$
}

Published online: 8 June 2017

(C) Springer Science+Business Media New York 2017

\section{Erratum to: J Supercomput DOI 10.1007/s11227-017-2064-0}

The original version of this article unfortunately contained a mistake. The section "Acknowledgements" was missing. The Acknowledgements section is given below.

Acknowledgements This work was supported by WCSL (World Class Smart Lab) research grant directed by Inha University. This work was also supported by Inha University Research Grant.

The online version of the original article can be found under doi:10.1007/s11227-017-2064-0.

$\triangle$ Wonik Choi

wichoi@inha.ac.kr

Boseon Yu

man82bs@gmail.com

Ling Liu

lingliu@cc.gatech.edu

1 Korea Institute of Science and Technology, Hwarang-ro 14gil 5, Seoul, Republic of Korea

2 School of Information and Communication Engineering, Inha University, 100 Inharo, Nam-Gu, Incheon, Republic of Korea

3 College of Computing, Georgia Institute of Technology, 266 Ferst Dr, Atlanta, GA, USA 